\title{
Modeling of the Optimal Flight Route \\ of Unmanned Aerial Vehicles Based \\ on Infrared Video Navigation Data Based \\ on the Upgraded Dijkstra Algorithm
}

\author{
Igor N. Ischuk ${ }^{\mathrm{a}}$ and Michael A. Likhachev ${ }^{\mathrm{b} *}$ \\ ${ }^{a}$ Siberian Federal University \\ Krasnoyarsk, Russian Federation \\ ${ }^{b}$ Military Training and Research Center of the Air Force \\ "Air Force Academy ft. Professor N. E. Zhukovsky \\ and Y.A. Gagarin» \\ Voronezh, Russian Federation
}

Received 24.06.2021, received in revised form 06.08.2021, accepted 21.10.2021

\begin{abstract}
The article presents a model for constructing the optimal flight route of unmanned aerial vehicles based on infrared video navigation data based on the upgraded Dijkstra algorithm. The method and algorithm for implementing the model for constructing the optimal flight route are considered. Numerical estimates of the frequency distribution of correlation values between reference (reference) and current images in the infrared wavelength range at different times of the day are obtained. The actual time period of the day is determined for the creation and further use of reference (reference) images with the longest possible use time during the next day. Optimal routes are constructed with the coordinates of the reference (reference) route, relative to the time of the formation of the reference route, taking into account the recognition of the zones of blocking flights of unmanned aerial vehicles according to the Dijkstra algorithm.
\end{abstract}

Keywords: unmanned aerial vehicle, video navigation, Dijkstra algorithm, infrared images.

Citation: Ischuk I. N., Likhachev M. Modeling of the optimal flight route of unmanned aerial vehicles based on infrared video navigation data based on the upgraded dijkstra algorithm, J. Sib. Fed. Univ. Eng. \& Technol., 2021, 14(7), 788-802. DOI: $10.17516 / 1999-494 X-0356$

(C) Siberian Federal University. All rights reserved

This work is licensed under a Creative Commons Attribution-Non Commercial 4.0 International License (CC BY-NC 4.0).

* Corresponding author E-mail address: tuborg051@mail.ru 


\title{
Моделирование оптимального маршрута полета
}

\section{беспилотных летательных аппаратов}

по данным инфракрасной видеонавигации

на основе модернизированного алгоритма Дейкстры

\author{
И. Н. Ищук ${ }^{\text {a }}$ М.А. Лихачев \\ ${ }^{a}$ Сибирский федеральный университет \\ Российская Федерация, Красноярск \\ ${ }^{6}$ Военный учебно-научный иееттр Военно-воздушных сил \\ «Военно-воздушная академия имени профессора \\ Н. Е. Жуковского и Ю. А. Гагарина» \\ Российская Федерация, Воронеж
}

\begin{abstract}
Аннотация. В статье представлена модель построения оптимального маршрута полета беспилотных летательных аппаратов по данным инфракрасной видеонавигации на основе модернизированного алгоритма Дейкстры. Рассмотрен способ и алгоритм реализации модели построения оптимального маршрута полета. Получены численные оценки распределения частоты значений корреляции между опорными (эталонными) и текущими изображениями в инфракрасном диапазоне длин волн в разное время суток. Определен актуальный период времени суток для создания и дальнейшего применения опорных (эталонных) изображений с максимально длительным временем использования в течение следующих суток. Построены оптимальные маршруты с координатами опорного (эталонного) маршрута относительно времени формирования опорного маршрута с учетом распознавания зон блокирования полетов беспилотных летательных аппаратов по алгоритму Дейкстры.
\end{abstract}

Ключевые слова: беспилотный летательный аппарат, видеонавигация, алгоритм Дейкстры, инфракрасные изображения.

Цитирование: Ищук, И.Н. Моделирование оптимального маршрута полета беспилотных летательных аппаратов по данным инфракрасной видеонавигации на основе модернизированного алгоритма Дейкстры / И.Н. Ищук, М. А. Лихачев // Журн. Сиб. федер. ун-та. Техника и технологии, 2021, 14(7). С. 788-802. DOI: 10.17516/1999-494X-0356

\section{Введение}

Стремительное развитие современных технологий в таких областях, как аэродинамика, компьютерные технологии, электроника, робототехника, инерциальных, оптических и спутниковых навигационных систем, а также результаты синтеза композитных материалов позволили выйти на качественно новый уровень в создании комплексов с беспилотными летательными аппаратами (БпЛА). Беспилотные летательные аппараты имеют большое значение в стратегическом плане как в промышленных, так и в оборонных сферах развития любого государства. Логистика применения высокотехнологичных робототехнических систем и комплексов - важный принцип их качественного использования, что значительно снижает трудозатраты, затраты на ресурсы и, как следствие, стоимость использования, а это ведет к получению максимально выгодного результата. Одним из этих факторов при использовании БпЛА является устойчивая навигация в условиях воздействия помех на работу глобальных систем спутниковой навигации и построение оптимального маршрута полета с учетом заданных 
условий местности выполнения задания [1]. Решение задачи осуществления видеонавигации и построения оптимального маршрута в инфракрасном (ИК) диапазоне длин волн позволит повысить качество и надежность применения БпЛА с учетом условий местности выполнения полета [2-4]. Для построения оптимального маршрута в темное время суток и осуществления по нему полета БпЛА, а также мини(микро)-БпЛА предварительно разрабатывается полетная карта в ИК-диапазоне длин волн, зависящая от целого комплекса условий, одним из которых являются теплофизические параметры объектов воздушного мониторинга: на карте задаются конкретные координаты маршрута [5, 6].

\section{1. Постановка задачи}

Представим полетную карту в виде взвешенного ориентированного графа G(V, E) без дуг отрицательного веса как прямоугольную координатную сетку со сторонами $i \in(1, N)$ и $j \in(1, M)$, в которой центр элементарного прямоугольника географически совпадает с соответствующей вершиной графа, образующих множество V. Каждая вершина графа, кроме граничных, соединена с соседними вершинами с помощью четырех ребер графа одинаковой длины, образующих множество ребер графа $\mathbf{E}$, для каждого из которых может быть задан свой вес:

$$
w(i, j)=\left\{\begin{array}{l}
(W, \text { if } K(F I R(t), F I R e(t)) \geq 1 / 2 \text { when }(i, j) \in U, \\
\infty, \text { unless }
\end{array}\right.
$$

значение которого $W$ определяется заданным классификатором, реализованным с помощью корреляционной функции $K(\cdot)$ [7]. Двумерный коррелятор осуществляет вычисление значения корреляционной функции $K(\cdot)$ по данным ИК-съемки в дальнем ИК-диапазоне FIR (3-5 мкм) с БпЛА в надир. Бортовой вычислитель БпЛА производит расчет коэффициента корреляции между набором ранее полученных для заданных моментов времени $t$ эталонных ИК-изображений $\operatorname{FIR}_{\mathrm{e}}\left(t^{*}\right)$ объектов классификатора $W$ и текущих ИК-изображений $\operatorname{FIR}(t)$, получаемых в ходе полета. Время получения эталонных изображений объектов классификатора $t^{*}$ определяется технологией построения тепловых томограмм [8]. При выполнении условия корреляционного критерия $K(\cdot) \geq 1 / 2$ формируется множество посещенных вершин $\mathbf{U}$.

Задача: необходимо на основе модернизации алгоритма Дейкстры найти кратчайший путь между заданными вершинами $a$ и $u$, несвязанного графа $G$, веса ребер которого $w$ вариативно изменяются во времени и зависят от значения корреляционной функции $K(\cdot)[9,10]$.

\section{2. Моделирование}

Для инициализации процесса моделирования полета БпЛА были заданы следующие условия:

1. Инфракрасные изображения района полетной карты известны и получены в летнее время для центральной европейской полосы России в моменты времени: 0:00, 2:00, 6:00, 8:00, 10:00, 12:00, 14:00, 16:00, 18:00, 20:00, 22:00 и 24:00.

2. Задана прямоугольная координатная сетка полетной карты со сторонами $N=M=20$, при этом кратчайший путь ищут между вершинами $a=(1,20)$ и $u=(2,2)$.

3. Множество классов $W$, определяющих веса ребер графа, задано и представлено в табл. 1.

$$
-790-
$$


Таблица 1. Множество классов $W$

Table 1. The set of $W$ classes

\begin{tabular}{|c|c|l|}
\hline $\begin{array}{c}\text { Номер } \\
\text { класса }\end{array}$ & $\begin{array}{c}\text { Значение веса } \\
\text { класса, W }\end{array}$ & \multicolumn{1}{c|}{ Описание класса } \\
\hline 1 & 1 & Открытый участок местности (равнина, ...) \\
\hline 2 & 2 & Техногенный участок местности (здания, сооружения и т. п.) \\
\hline 3 & & Участок местности с густой растительностью (лес, кустарник и т. п.) \\
\hline 4 & & Горный участок местности \\
\hline$\ldots$ & $\ldots$ & $\ldots$ \\
\hline 9 & 9 & Маловысотный комплекс (средство, система) противовоздушной обороны \\
\hline
\end{tabular}

4. Моделирование полета БпЛА осуществляется в двумерном пространстве при заданной постоянной высоте полета. Скорость путевого ветра при полете БпЛА принята равной нулю. В случае отсутствия данных о местоположении БпЛА от блока ИК-видеонавигации в введенной координатной сетке ориентация маршрута полета осуществляется по магнитному компасу $[11,12]$.

\section{А. Моделирование № 1. Маршруты полета БпЛА с учетом рельефа местности}

Моделирование полета БпЛА по маршруту с учетом рельефа местности включает два этапа:

Этап № 1 - построение эталонного (опорного) маршрута для ИК-изображений, полученных в 24:00, при этом изображение каждого элементарного прямоугольника соответствующей вершины графа считается известным, а значение веса каждого из четырех ребер задано по данным классификатора табл. 1. Применение алгоритма Дейкстры обеспечивает получение эталонного маршрута между заданными вершинами $a$ и $u$ (рис. 1a), обозначенного пунктирной линией малинового цвета. Длина оптимального маршрута в представленном численном эксперименте составляет 22 ребра. На основании условия для эталонного маршрута $K(\cdot)=1$ - модернизированный алгоритм, обеспечивающий расчет весов для каждого из ребер графа согласно (1) с формированием множество посещенных вершин $\mathbf{U}$, отраженного на рис. 1 выделенными элементарными прямоугольниками координатной сетки с границами зеленого цвета.

Этап № 2 - построение текущего маршрута по данным набора эталонных изображений на 24:00 и текущего времени полета (2:00, 6:00, 8:00, 10:00, 12:00, 14:00, 16:00, 18:00, 20:00, 22:00 и 24:00) (рис. $1 b-1)$.

В результате моделирования полета по ИК-видеонавигации были получены значения корреляции между текущими ИК-изображениями и эталонными (опорными); статистические оценки частоты этих значений корреляции относительно времени полета представлены на рис. 2.

При этом общее число эталонных изображений (массив эталонных изображений) в соответствии с введенной координатной сеткой составило 400 шт., а длина маршрута варьируется от 18 до 23 ребер графа, что обусловлено вероятностью распознавания объекта классификато- 


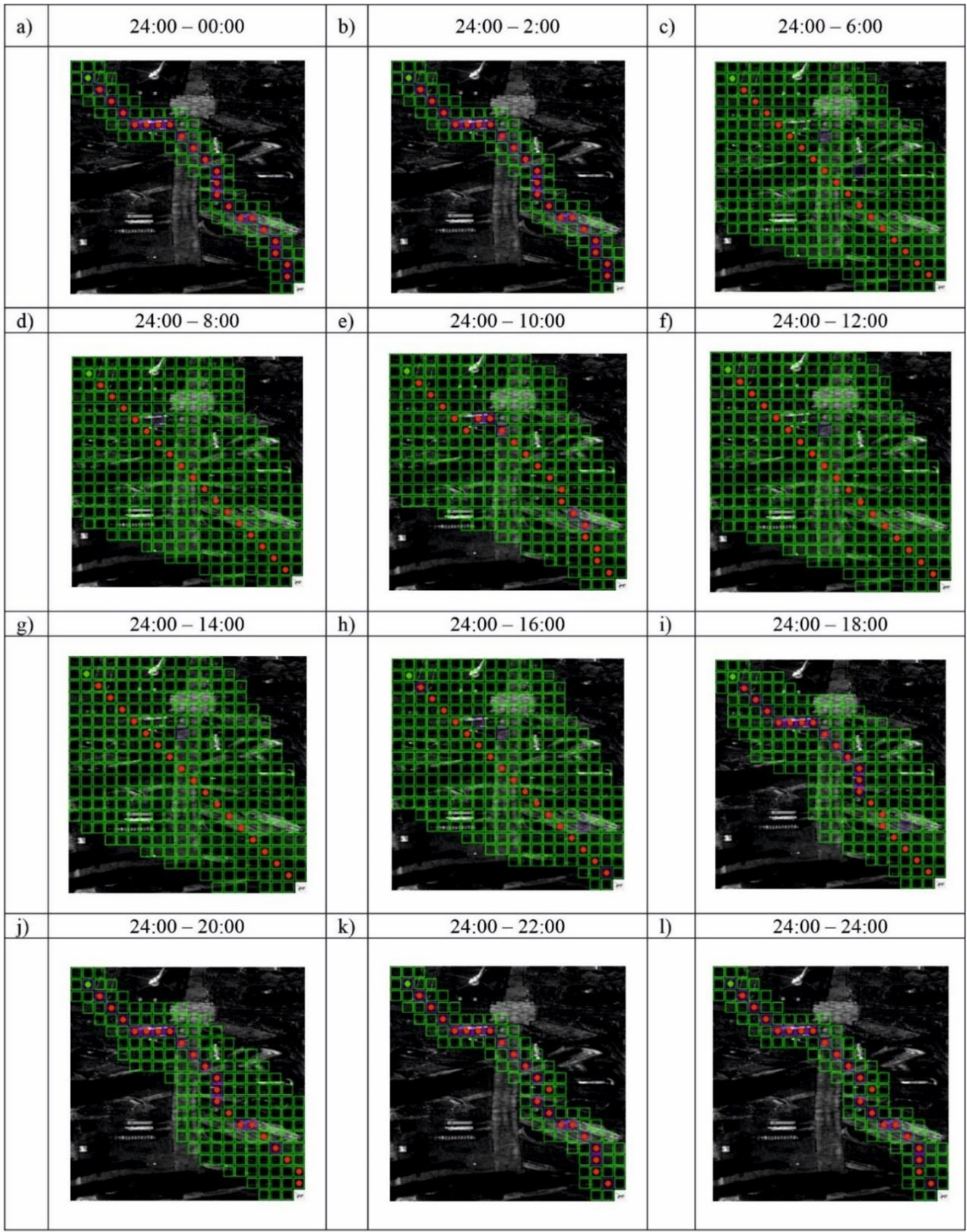

Рис. 1. Маршруты полета БпЛА: а - опорный (эталонный) маршрут на 24:00; b-1 - текущие маршруты, построенные по ИК-изображениям района полетов относительно опорного, полученные в 2:00, 6:00, 8:00, $10: 00,12: 00,14: 00,16: 00,18: 00,20: 00,22: 00$ и 24:00 соответственно

Fig. 1. UAV flight routes: a - reference route for 24: 00; b-1 - the current routes plotted from the IR images of the flight area relative to the reference one, obtained: in 2:00, 6:00, 8:00, 10:00, 12:00, 14:00, 16:00, 18:00, 20:00, 22:00 and 24:00, respectively 


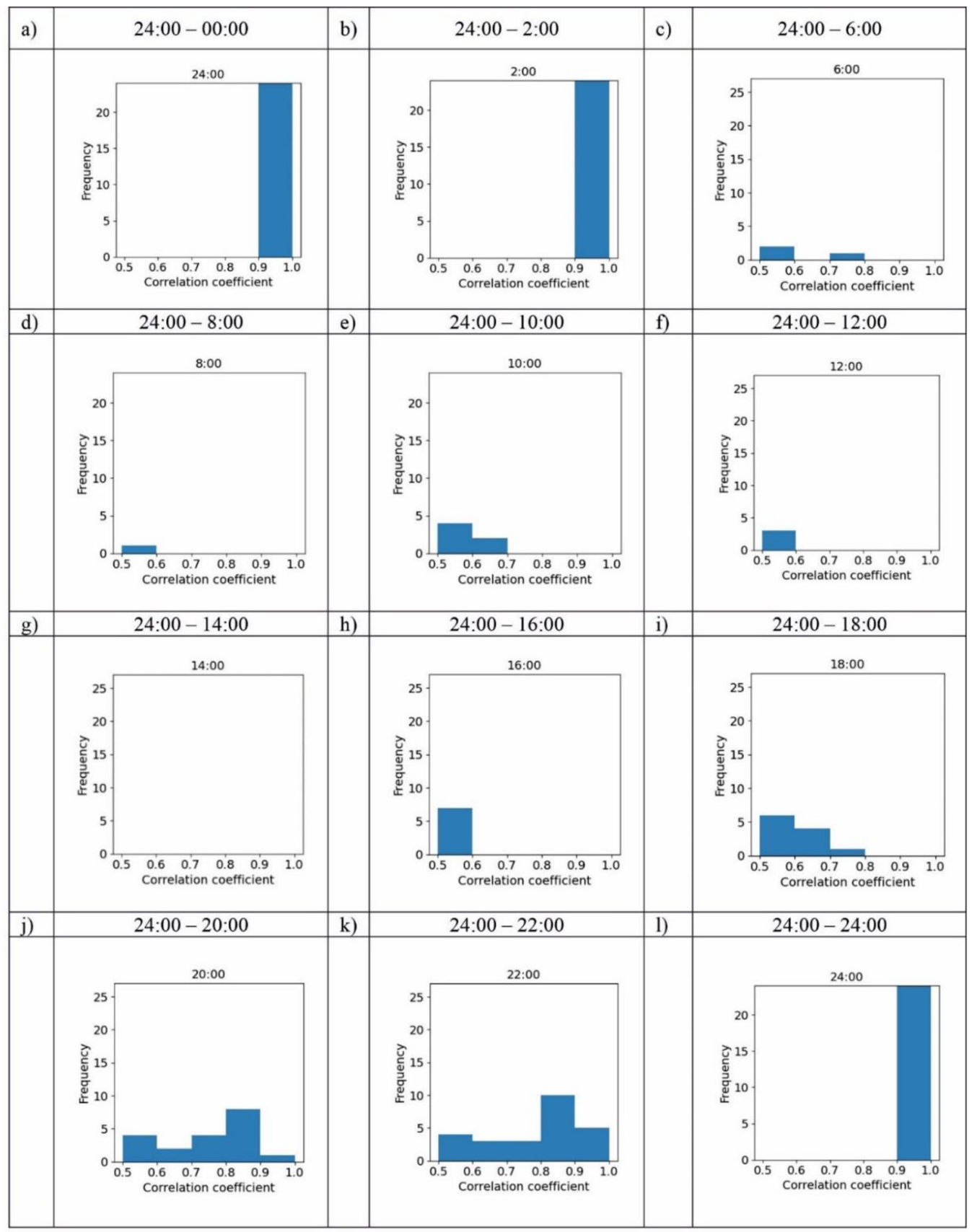

Рис. 2 Гистограмма распределения частоты полученных значений корреляции относительно времени полета для: а - эталонного маршрута на 24:00; b-1 - 2:00, 6:00, 8:00, 10:00, 12:00, 14:00, 16:00, 18:00, 20:00, 22:00, 24:00

Fig. 2. Histogram of the frequency distribution of the obtained correlation values relative to the flight time for: $\mathrm{a}$ - reference route for $24: 00 ; \mathrm{b}-1-2: 00,6: 00,8: 00,10: 00,12: 00,14: 00,16: 00,18: 00,20: 00,22: 00,24: 00$

ра. При $K(\cdot)<1 / 2$ вес ребра на основании (1) является $\infty$. В этом случае значение веса ребра графа алгоритмически задается числом, значение которого больше на одну единицу максимального значения, определенного в классификаторе. 
В результате «нераспознавания» ни одного из заданных эталонных (опорных) объектов «оптимальный» маршрут полета БпЛА между заданными вершинами $a$ и $u$ представляет собой прямую линию, что соответствует маршрутам, изображенным на рис. $1 c, d, f, g, h$, и продиктовано введением условия № 4 - скорость путевого ветра равна нулю, а ориентация маршрута полета осуществляется по магнитному компасу. При этом цвет линии (точек) маршрута, совпадающей с эталонным маршрутом, отображается малиновым цветом, в остальных случаях - красным.

\section{Б. Моделирование № 2. Маршруты полета БпЛА с учетом обхода районов блокирования полетов}

Моделирование полета БпЛА с учетом обхода районов блокирования также включает два этапа:

Этап № 1 - построение эталонного (опорного) маршрута для ИК-изображений, полученных в 24:00 с учетом обхода трех районов блокирования полетов на основе алгоритма Дейкстры между заданными вершинами $a$ и $u$ (рис. $4 a$ ), обозначенного пунктирной линией красного цвета [13]. Длина оптимального маршрута составляет 26 ребер. Заданные районы блокирования полетов выделены элементарными прямоугольниками координатной сетки с границами фиолетового цвета [14]. В ходе полета БпЛА блок ИК-видеонавигации по данным текущих ИК-изображений, получаемых на расстоянии не менее длины одного ребра графа $G$, рассчитывает значение коэффициента корреляции. Длина одного ребра графа равна расстоянию между текущим местоположением БпЛА и центром координат анализируемого элементарного прямоугольника. Условием для распознавания элементарного прямоугольника района блокирования полетов является корреляционный критерий $K(\cdot) \geq 1 / 2$.

Этап № 2 - построение текущего маршрута по данным эталонных набора изображений на 24:00 и текущего времени полета $(2: 00,6: 00,8: 00,10: 00,12: 00,14: 00,16: 00,18: 00,20: 00,22: 00$ и 24:00) (рис. $3 b-1)$.

В результате моделирования полета с учетом обхода районов блокирования по ИКвидеонавигации были получены значения корреляции между текущими ИК-изображениями и эталонными (опорными), статистические оценки частоты полученных значений корреляции относительно времени полета представлены на рис. 4. В ходе моделирования «нераспознанные» элементарные прямоугольники районов блокирования отображены с границами оранжевого цвета.

\section{3. Анализ результатов моделирования}

Анализ полученных данных показывает, что с увеличением разницы по времени между текущим и эталонным ИК-изображениями значение коэффициента корреляции уменьшается. В результате моделирования процесса полета БпЛА с построением маршрута для ИК-изображений, полученных в 2:00, 6:00, 8:00, 10:00, 12:00, 14:00, 16:00, 18:00, 20:00, 22:00 и 24:00 местного времени, были получены частоты распределения значения коэффициента корреляции в течение суток. На рис. 5 и 6 представлены гистограммы распределения частоты совпадений координат текущего маршрута с координатами опорного (эталонного) маршрута относительно местного времени формирования опорного маршрута при отсутствии и наличии районов блокирования полетов соответственно. 


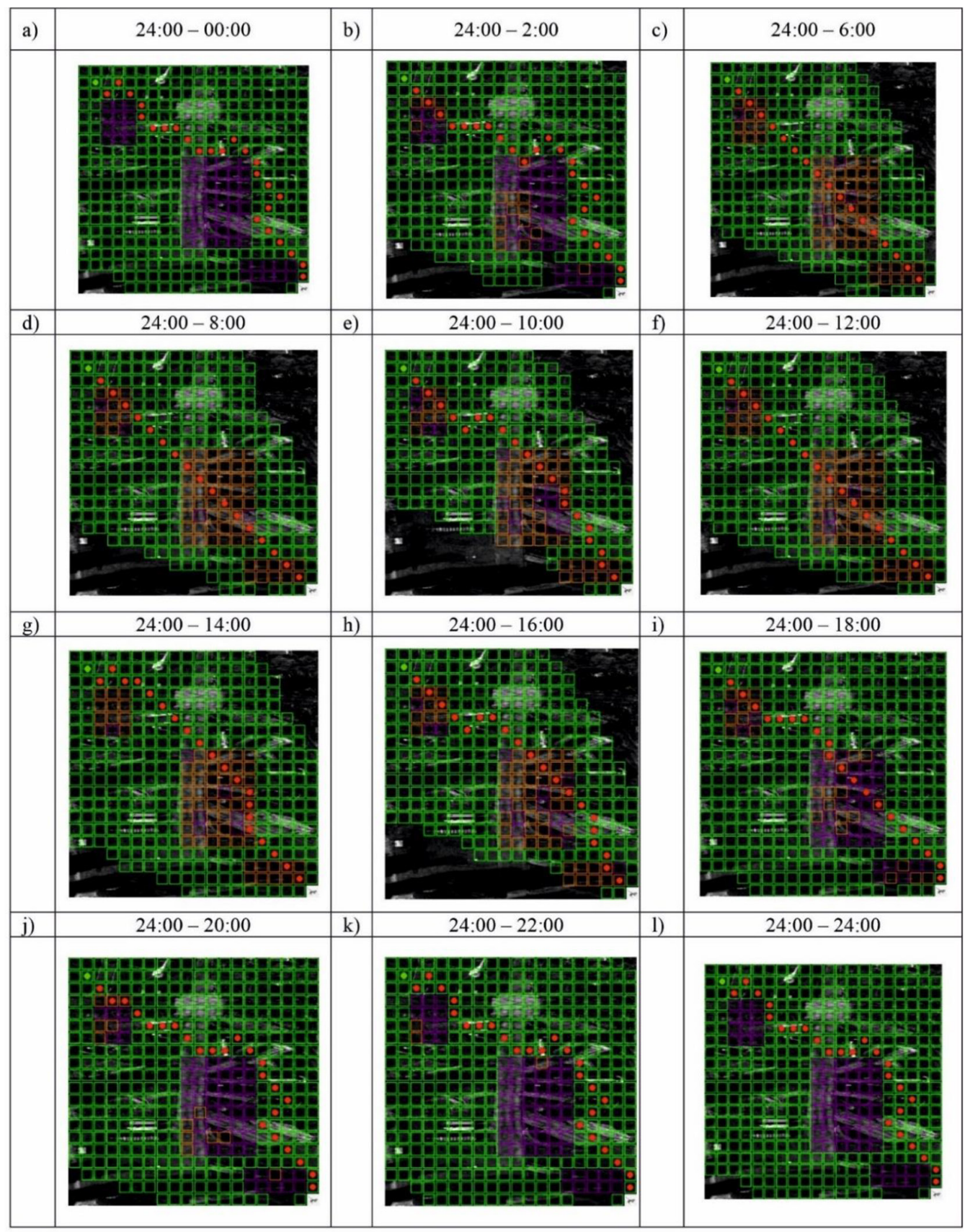

Рис. 3. Маршруты полета БпЛА с учетом обхода районов блокирования полетов: А - опорный (эталонный) маршрут на 24:00; b-1 - текущие маршруты, построенные по ИК-изображениям района полетов относительно опорного, полученные в 2:00, 6:00, 8:00, 10:00, 12:00, 14:00, 16:00, 18:00, 20:00, 22:00 и 24:00 соответственно

Fig. 3. UAV flight routes, taking into account the circumvention of flight blocking areas: A - reference (reference) route for 24:00; b-1 - the current routes plotted from the IR images of the flight area relative to the reference one, obtained: in 2:00, 6:00, 8:00, 10:00, 12:00, 14:00, 16:00, 18:00, 20:00, 22:00 and 24:00, respectively 


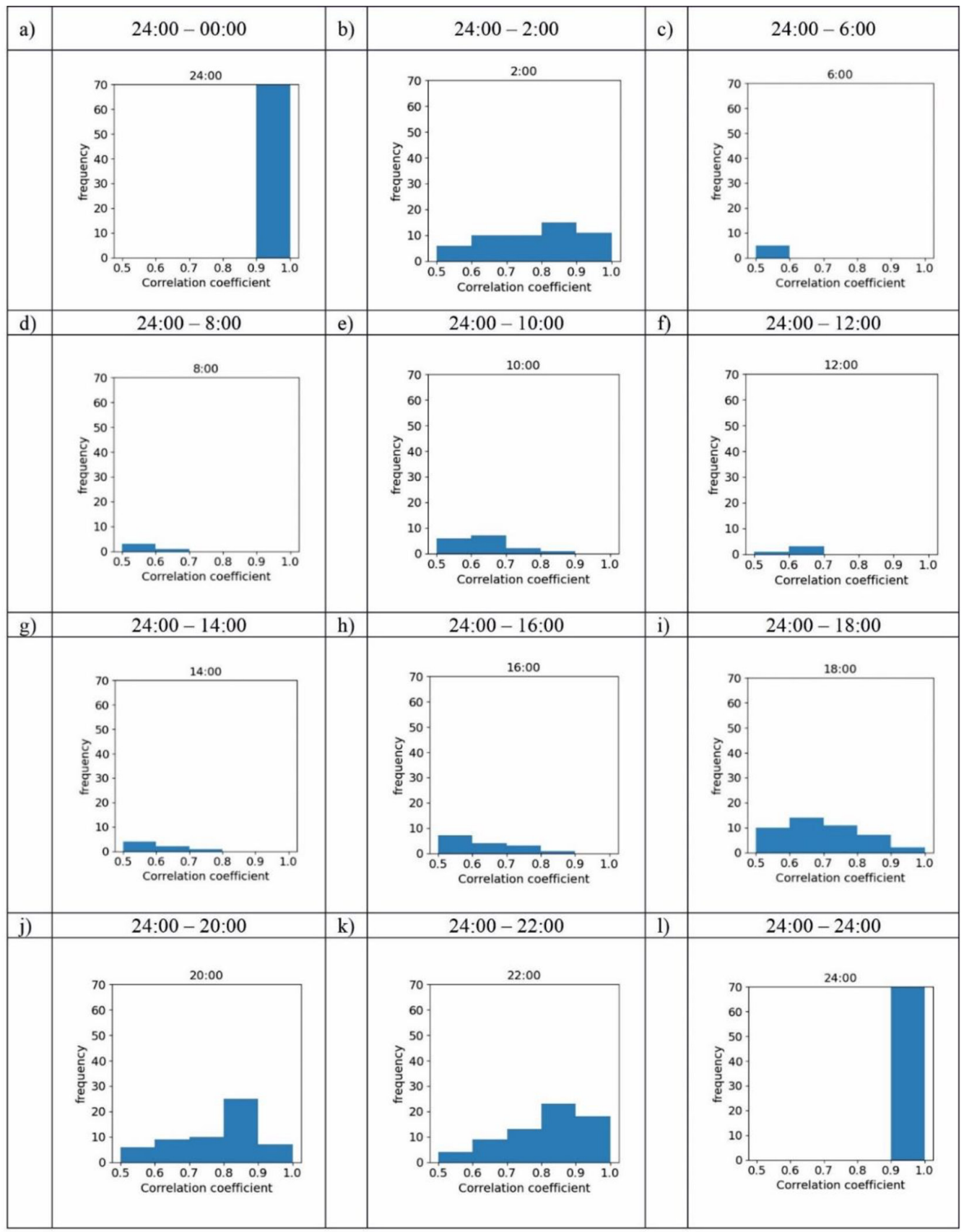

Рис. 4. Гистограмма распределения частоты полученных значений корреляции относительно времени полета для: а - эталонного маршрута с учетом зон блокирования полетов на 24:00; b-1 - 2:00, 6:00, 8:00, 10:00, 12:00, 14:00, 16:00, 18:00, 20:00, 22:00, 24:00

Fig. 4. Histogram of the frequency distribution of the obtained correlation values relative to the flight time for: $\mathrm{a}-$ the reference route, taking into account the flight blocking zones at 24: 00; b-1 - 2:00, 6:00, 8:00, 10:00, 12:00, 14:00, 16:00, 18:00, 20:00, 22:00, 24:00 
Histogram of the distribution of the frequency of coincidences of relative to the astronomical time of the formation of the reference route
resoute.

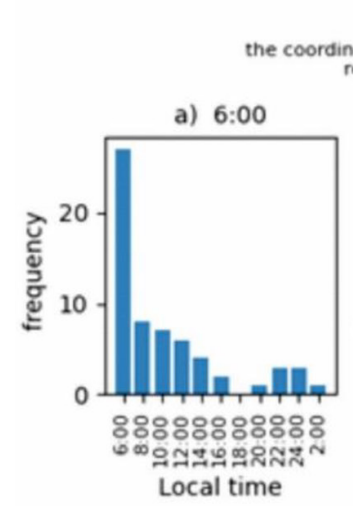

a) 6:00

e) 14:00

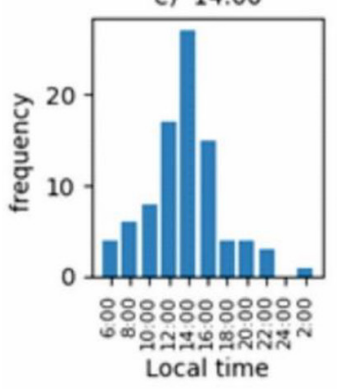

i) $22: 00$

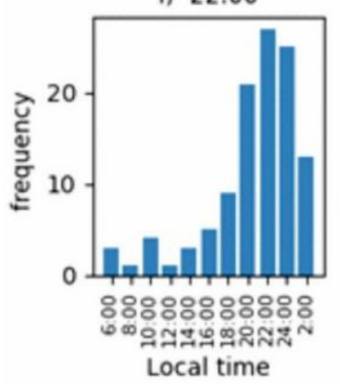

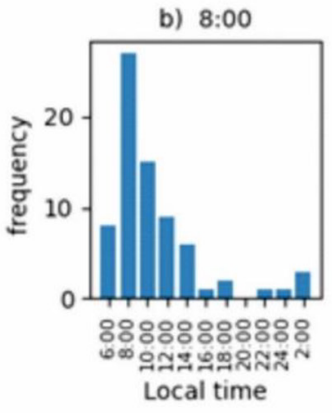

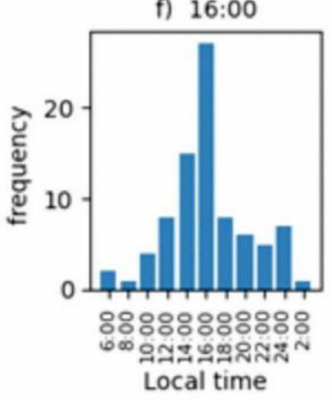

j) $24: 00$

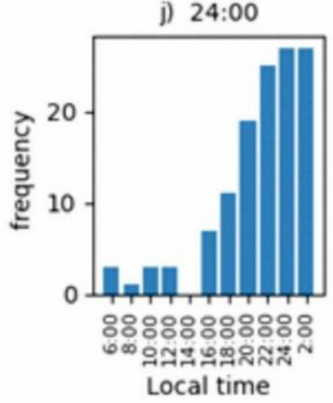

c) 10:00

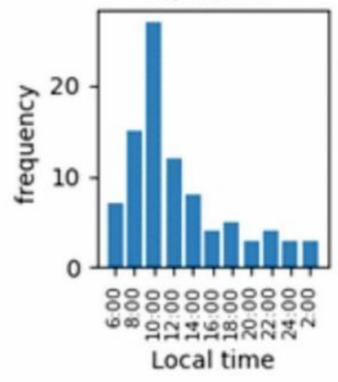

g) $18: 00$

d) $12: 00$

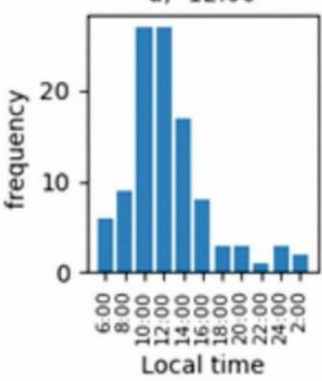

h) 20:00
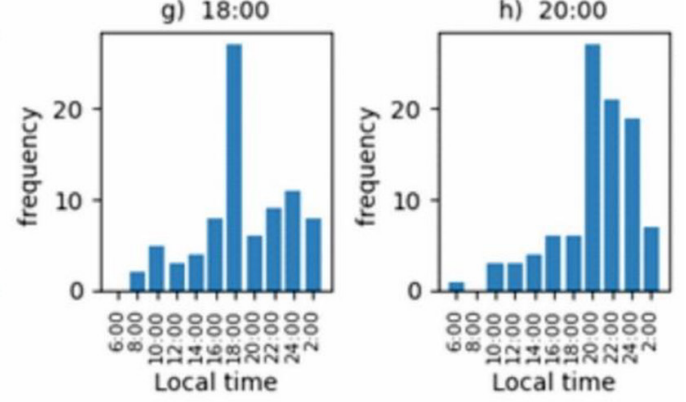

Рис. 5. Гистограмма распределения частоты совпадений координат текущего маршрута с координатами опорного (эталонного) маршрута относительно времени формирования опорного маршрута

Fig. 5. Histogram of the distribution of the frequency of coincidences of the coordinates of the current route with the coordinates of the reference (reference) route, relative to the time of formation of the reference route

На основании полученных распределений произведена оценка вероятности нахождения

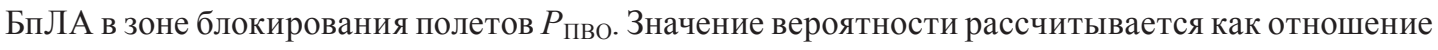
частоты попадания летательного аппарата в элементарный прямоугольник зоны блокирования к значению всех возможных состояний. Соответственно, вероятность обхода зон блокирования полетов составит $P(\mathrm{t})=1-P_{\text {пво }}(t)$. Графики зависимости вероятности обхода зон блокирования полетов от относительного времени $\mathrm{P}(\Delta t)$, где $\Delta t=t-t^{*}$, представлены на рис. 7.

Результаты оценок частоты распознавания местоположения БпЛА по данным массива эталонных изображений элементарных прямоугольников района полетов (по 400 изображений на каждый из 11 моделируемых интервалов времени полетов в течение одних суток) с учетом обхода зон блокирования полетов (районов противовоздушной обороны (ПВО) противника) представлены на рис. 8 . 


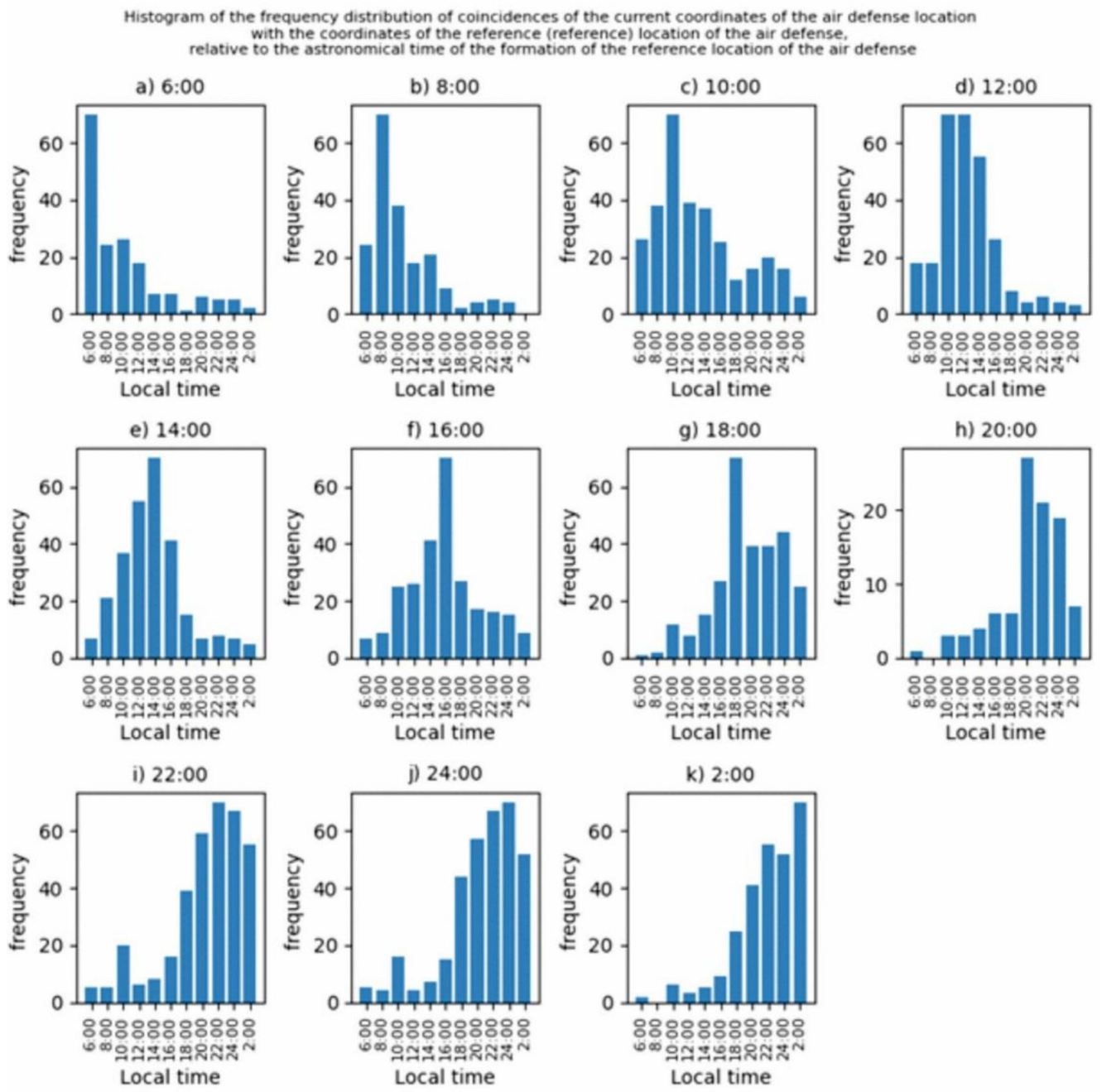

Рис. 6. Гистограмма распределения частоты совпадений координат текущего маршрута с координатами опорного (эталонного) маршрута относительно времени формирования опорного маршрута с учетом распознавания зон блокирования полетов БпЛА

Fig. 6. Histogram of the distribution of the frequency of coincidences of the coordinates of the current route with the coordinates of the reference (reference) route, relative to the time of formation of the reference route, taking into account the recognition of UAV flight blocking zones

Из анализа графиков рис. 8 следует, что эталонные изображения, сформированные для 22:00 и 24:00, при пролонгации параметров погоды на следующие сутки характеризуются наибольшими значениями частоты распознавания местоположения БпЛА по данным ИК-видеонавигации. При этом 22- и 24-часовые эталонные (опорные) изображения обеспечивают возможность распознавания и обхода зон блокирования полетов с вероятностью не ниже 0.8 на интервале времени от 20:00 до 4:00, а следовательно, выполнение задачи ИК-видеонавигации на протяжении 6 ч полетов БпЛА в условиях сумерек и ночи.

При проведении полетов днем эталонные (опорные) ИК-изображения могут находиться в актуализированном состоянии на интервале времени, не превышающем двух часов. 


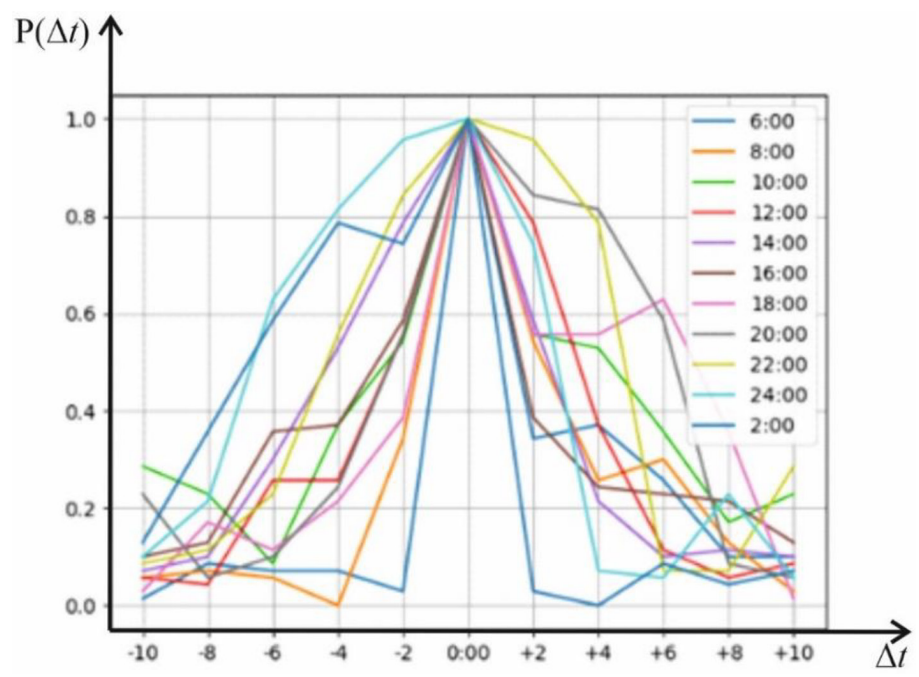

Рис. 7. Графики зависимости вероятности обхода зон блокирования полетов по данным ИКвидеонавигации относительно времени формирования ИК опорного (эталонного) изображения района полетов

Fig. 7. Graphs of the dependence of the probability of bypassing the flight blocking zones according to the IR video navigation data, relative to the time of formation of the IR reference (reference) image of the flight area
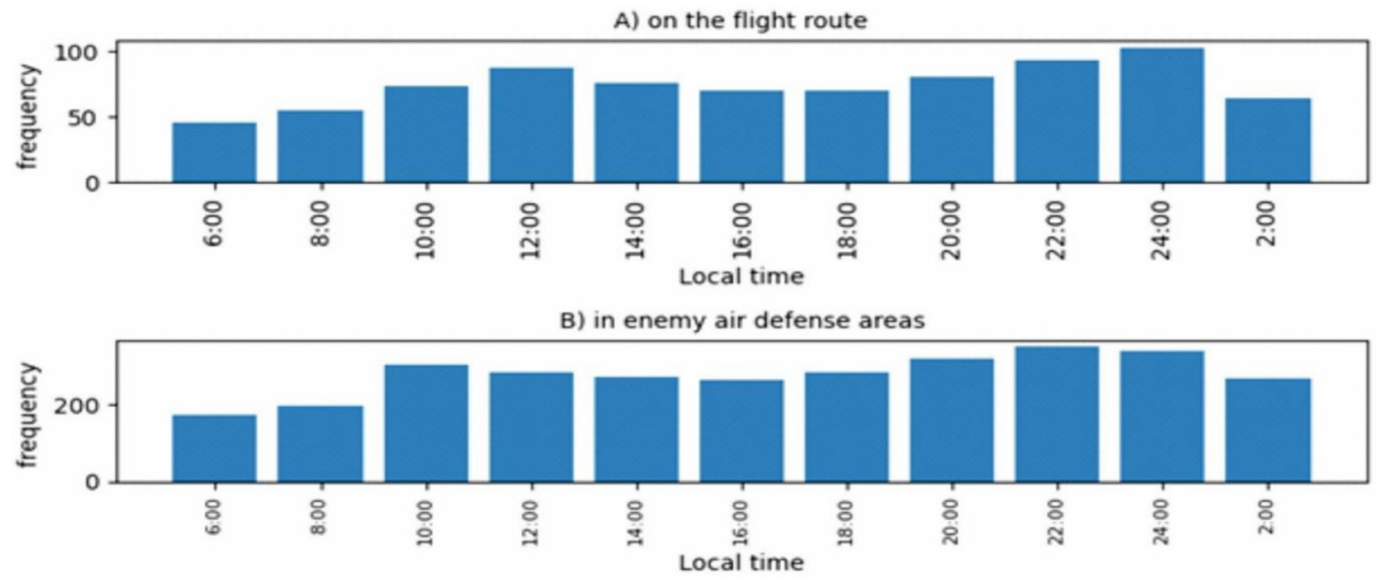

Рис. 8. Гистограмма частоты распознавания местоположения БпЛА

Fig. 8. Histogram of the frequency of UAV location recognition

\section{Вывод}

В системах автономной видеонавигации БпЛА в ночных условиях для использования в качестве эталонных (опорных) ИК-изображений (ортофотопланов) наиболее эффективными являются изображения, сформированные через 1-2 ч после заката и за 1 ч до восхода солнца.

\section{Список литературы / References}

[1] Коровин А.В., Миронов В.А., Новиков А. А., Трущинский А. Ю., Фатеев Ю. Л. Методика оценки помехоустойчивости перспективного навигационного приемника ГНСС беспилотно- 
го летательного аппарата. Журн. Сиб. федер. ун-та. Техника и технологии, 2016, 9(8), 1162-1171 [Korovin A. V., Mironov V.A., Novikov A. A., Trushchinskij A. Yu., Fateev Yu.L. Methodology for assessing the noise immunity of a promising navigation GNSS receiver of an unmanned aerial vehicle. Journal Sib. feder. un-ta. Engineering and Technologies, 2016, 9(8), 1162-1171 (in Russian)]

[2] Веселов Ю.Г., Данилин А.А., Тихонычев В.В. Выбор тест-объекта для оценки разрешающей способности цифровых оптико-электронных систем мониторинга земной поверхности. Наука и образование, 2012, 4 [Veselov Yu.G., Danilin A. A., Tikhonichev V. V. Select the test object for evaluation of the resolution of the digital opto-electronic systems for monitoring the earth's surface. Science and education, 2012, 4 (in Russian)]

[3] Свищо В.С., Парфильев А.В., Гарин Е.Н. Обработка динамических инфракрасных изображений при посадке беспилотных летательных аппаратов самолетным способом для контроля навигационных параметров. Журн. Сиб. федер. ун-та. Техника и технологии, 2016, 9(3), 392-400 [Svishcho V.S., Perfiliev A. V., Garin E. N. Processing of dynamic infrared images during the landing of unmanned aerial vehicles by an airplane method for monitoring navigation parameters. Journal Sib. feder. un-ta. Engineering and Technologies, 2016, 9(3), 392-400 (in Russian)]

[4] Ищук И.Н., Долгов А.А., Филимонов А.М., Дмитриев Д.Д. Методика оценки динамики изменения вероятностных характеристик распознавания объектов воздушной разведки. Журн. Сиб. федер. ун-та. Техника и технологии, 2019, 12(6), 683-693 [Ishchuk I.N., Dolgov A. A., Filimonov A. M., Dmitriev D. D. Methodology for assessing the dynamics of changes in the probabilistic characteristics of recognition of objects of aerial reconnaissance. Journal Sib. feder. un-ta. Engineering and Technologies, 2019, 12(6), 683-693 (in Russian)]

[5] Ищук И.Н., Долгов А.А., Лихачев М.А., Тельных Б.К. Модель расчета теплофизических параметров материалов по данным многоспектральной разновременной фотографической съемки земной поверхности. Журн. Сиб. федер. ун-та. Техника и технологии, 2020, 13(7), 906-918 [Ishchuk I. N., Dolgov A. A., Likhachev M.A., Telnykh B.K. Model of calculation of thermophysical parameters of materials based on multispectral multi-time photographic survey of the Earth's surface. Journal Sib. Feder. un-ta. Engineering and Technology, 2020, 13(7), 906-918 (in Russian)]

[6] Пат. 2707387 C1 Российская Федерация, МПК G01J5/00. Способ дистанционного определения пространственного распределения теплофизических параметров земной поверхности/ Ищук И.Н., Долгов А. А.; заявитель и патентообладатель Федеральное государственное казенное военное образовательное учреждение высшего образования «Военный учебно-научный центр Военно-воздушных сил «Военно-воздушная академия имени профессора Н.Е. Жуковского и Ю.А. ВУНЦ ВВС «ВВА», Гагарина» (г. Воронеж) Министерства обороны Российской Федерации (RU) № RU2707387C1; заявл. 28.02.2019; опубл. 26.11.2019, 11 с. [PAT. 2707387 C1 of the Russian Federation, IPC G01J5 / 00. Method for determination of the spatial distribution of thermophysical parameters of the earth's power/ Ishchuk I. N., Dolgov A. A.; applicant and tattoobilder Federal state military educational higher education «Military training and research centre of Militaryair forces «Military-air Academy named after Professor N.E. Zhukovsky and Y. A. VUNTS VVS «VVA» Gagarin» (Voronezh) of the Ministry of defense of the Russian Federation (ru) No. ги2707387c1; Appl. 28.02.2019; opubl. 26.11.2019, 11 P. (in Russian)] 
[7] Парфирьев А.В. Модель системы автоматического сопровождения. Актуальные проблемы и перспективные направления развития комплексов авиационного оборудования. Сб. науч. ст. по материалам V Международной науч.-практ. конф. «Академические Жуковские чтения» (22-23 ноября 2017 г.): тез. докл. Воронеж: ВУНЦ ВВС ВВА, 2017. С. 112-116 [Porfiriev A. V. Model of the automatic support system. Actual problems and promising directions of development of aviation equipment complexes. Collection of scientific articles based on the materials of the V International Scientific and Practical Conference «Academic Zhukovsky Readings» (November 22-23, 2017): collection of mat.crystals proc. dokl. Voronezh: VUNTS VVS VVA, 2017. P. 112-116 (in Russian)]

[8] Ищук И.Н., Филимонов А.М., Долгов А.А., Степанов Е.А. Алгоритм совместной обработки многоспектральных изображений по данным воздушной съемки с беспилотных летательных аппаратов. Промышленные АСУ и контроллеры, 2018, 10, 27-34 [Ishchuk I.N., Filimonov A. M., Dolgov A. A., Stepanov E. A. Algorithm of joint processing of multispectral images based on aerial survey data from unmanned aerial vehicles. Industrial automated control systems and controllers, 2018, 10, 27-34 (in Russian)]

[9] Басараб М.А., Домрачева А.Б., Купляков В.М. Алгоритмы решения задачи быстрого поиска пути на географических картах. Инженерный журнал: наука и инноваиии, 2013, 11, 2-12 [Basarab M.A., Domracheva A. B., Kuplyakov V. M. Algorithms for solving the problem of fast path search on geographical maps. Engineering Journal: Science and Innovation, 2013, 11, 2-12 (in Russian)]

[10]Копылов М.Д., Хохлов К.А. Поиск кратчайших путей в транспортных сетях. Научно-образовательный журнал для студентов и преподавателей «StudNet», 2021, 4(5), 1-16 [Kopylov M.D., Khokhlov K. A. Search for shortest paths in transport networks. Scientific and educational journal for students and teachers «StudNet». 2021, 4(5), 1-16 (in Russian)]

[11] Бондарев В.Г. Видеонавигация летательного аппарата. Компьютерные и информационные науки, Электротехника, электронная техника, информационные технологии, Механика и машиностроение, Технологии материалов, 2015, 213, 65-72 [Bondarev V.G. Video navigation of an aircraft. Computer and information sciences, Electrical engineering, electronic engineering, information technologies, Mechanics and mechanical engineering, Materials technologies, 2015, 213, 65-72 (in Russian)]

[12]Семенова Л. Л. Современные методы навигации беспилотных летательных аппаратов. Наука и образование сегодня, 2018, 4(27), 6-8 [Semenova L. L. Modern methods of navigation of unmanned aerial vehicles. Science and education today, 2018, 4(27), 6-8 (in Russian)]

[13]Абрамянц Т.Г. Е.П. Маслов, И. М. Рудько, В.П. Яхно. Уклонение подвижного объекта от обнаружения группой наблюдателей при малых отношениях сигнал/помеха. Информационно-управляющие системы, 2011, 2(51), 2-7 [Abramyants T.G., Maslov E.P., Rudko I. M., Yakhno V.P. Evasion of a moving object from detection by a group of observers at a small signal/interference ratio. Information and control Systems, 2011, 2(51), 2-7 (in Russian)]

[14] Абраменков В.В., Васильченко О. В., Муравский А. П. Обоснование подхода к построению системы селекции движущихся целей радиолокационной станции обнаружения малоразмерных беспилотных летательных аппаратов. Журн. Сиб. федер. ун-та. Техника и технологии, 2019, 12(7), 780-791 [Abramenkov V. V. Vasilchenko O. V., Muravsky A. P. Substantiation of an

$$
-801-
$$


approach to the construction of a system for selecting moving targets of a radar station for detecting small-sized unmanned aerial vehicles. Journal Sib. feder. un-ta. Engineering and Technologies, 2010, 12(7), 780-791 (in Russian)] 